\title{
S54. Expert judgement on the use of psychotherapeutic medication
}

\section{CONCEPTS AND METHODS \\ MB Balter*. TA Ban. EH Uhlenhuth \\ Public Health Research Center, Inc \\ 2822 Connecticut Ave NW \\ Washington DC 20008 USA.}

An international study of expert judgement and opinion about the use of benzodiazepines and other psychotherapeutic medications was conducted with the purpose (a) to collect and render accessible the valuable information and wisdom that personal experience can provide about drug treatment (b) to generate information that might help optimize current practice or suggest new clinical applications for available medications and (c) to accomplish above in a systematic quantitative manner.

A panel of psychiatrists with internationally recognized expertise in clinical psychopharmacology and pharmacotherapy was constructed by a peer nomination process. It included 73 Experts from 25 countries named by at least 2 of the original nominators or by those already selected for the Panel in an iterative process until no new names appeared. The Expert Panel provided data on their familiarity with psychotherapeutic medications, indications for the use of these medications, choice of initial treatments in specific clinical circumstances, pharmacological considerations in the treatment of psychiatric disorders with special emphasis on dependence and abuse liability, therapeutic equivalence of various benzodiazepines and nonbenzodiazepines in the treatment of psychiatric disorders, judgements on selected controversial therapeutic propositions and judgements on certain drug regulatory policies and actions.

The data were collected by means of a self-administered questionnaire, sent in the mail. There was no prior communication with the Experts. The response rate was $90.4 \%(66 / 73)$.
RANGE OF MEDICATIONS RECOMMENDED FOR TREATMENT T.A. Ban, M.B. Balter, and E.H. Uhlenhuth

Department of Psychiatry, Vanderbilt University, Nashville, TN 37232 , USA

This report, from a survey of judgments by a Panel of internationally recognized Experts, focuses on the range of medications recommended by the Expert Panel for the treatment of 5 sleep disorders, 3 adjustment disorders, 18 neurotic disorders, 14 substance induced disorders and organic delirium. In one section of the survey, the Experts were asked to identify their first and second choices of medication in each condition for which pharmacological treatment was indicated in their judgment. They were also asked specifically whether they would consider a benzodiazepine. More than half the Experts recommended a medication in 29 of the 41 conditions. In 8/29 disorders, more Experts recommended a benzodiazepine than a non-benzodiazepine. In the remaining 21/29 disorders, most of the Experts who recommended medication thought that a benzodiazepine merited consideration. Thus the Expert Panel judged benzodiazepines to have a very broad range of indications.

To provide a context for these recommendations, another section of the survey asked the Experts to rate their familiarity with 242 chemically distinct psychotherapeutic medications listed alphabetically by their generic and proprietary names, on the basis of personal clinical use, the literature, and other sources. None of the 66 Experts rated themselves as familiar or very familiar with $<20 \%$ of the medications. Sixteen rated themselves as familiar or very familiar with $20-39 \%$ of the medications, 40 with $40-59 \%$, 9 with $60-79 \%$, and one with $>79 \%$ of the medications. Thus the Experts' frequent recommendation of a benzodiazepine occurred in the context of familiarity with a wide variety of psychotherapeutic compounds.

*Deceased 
FACTORS INFLUENCING TREATMENT DECISIONS IN ANXIETY DISORDERS

E.H. Uhlenhuth, M.D., M.B. Balter, Ph.D., and T.A. Ban, M.D. University of New Mexico, Department of Psychiatry, 2400 Tucker, NE, Albuquerque, NM 87131, USA

This report, from a survey of judgments by a Panel of internationally recognized Experts, focuses on some clinical features that may affect choice of strategy in the pharmacotherapy of anxiety disorders. The survey included typical case vignettes of anxiety disorders followed by questions about therapeutic options. The data for this report were drawn from vignettes depicting contrasting conditions of functional impairment, duration of illness, use of alcohol, cardiac conduction status, and life stress.

Most Experts recommended a psychosocial procedure for initial treatment of agoraphobia, social phobia, and OCD irrespective of functional impairment, but few chose treatment for adjustment disorder without impairment. Most Panelists also favored medication in all 4 cases, except for social phobia and adjustment disorder without impairment. For panic, most Panelists recommended both psychotherapy and medication irrespective of the duration of illness. Most Experts chose both treatments for GAD, taking only slight account of current heavy alcohol use. Most recommended medication for OCD even with cardiac conduction deficit. Only a small majority recommended psychosocial treatment or medication for adjustment disorder with mild life stress.

The therapeutic recommendations of the Expert Panelists imply that anxiety disorders generally are serious illnesses that merit vigorous psychosocial and pharmacological intervention. Their recommendations also imply that the benefit/risk ratio of pharmacotherapy is most favorable in circumstances of high functional impairment, absent history of heavy alcohol use in GAD, normal cardiac conduction in $\mathrm{OCD}$, and marked life stress in adjustment disorder.
FACTORS INFLUENCING DISCONTINUATION OF MEDICATION M.B. Balter, Ph.D., E.H. Uhlenhuth, M.D., and T.A. Ban, M.D. University of New Mexico, Department of Psychiatry, 2400 Tucker, NE, Albuquerque, NM 87131, USA

This report, from a survey of judgments by a Panel of internationally recognized Experts, focuses on indications of therapeutic dose dependence with long term use of benzodiazepines (BZs) and their clinical significance in discontinuing medication. One section of the survey was devoted to specific questions concerning the relative risk, among BZs, of therapeutic dose dependence, withdrawal symptoms, difficulties in discontinuing medication, and the relative importance of a wide variety of factors in contributing to these problems.

The Experts most often identified longer duration of treatment, higher dose, shorter drug half life, predisposing personality, and past history of substance abuse or dependence as important factors increasing the probability of withdrawal symptoms. Only the first 2 factors were named by at least half the Experts. A vast majority thought that the intensity of withdrawal symptoms is greatly reduced and the differences between BZs with short and long half lives become clinically negligible when doses are tapered very gradually. A large majority also thought that patients with anxiety disorders unresponsive to other treatments should not be denied BZs despite a past history of substance abuse or dependence. The Experts most often named persistent anxiety, chronicity of the disorder, severity of the disorder, and predisposing personality as important factors in failure to achieve complete discontinuation of BZs. None of these, however, was identified by as many as half the Experts. The judgments of the Expert Panelists imply that pharmacological differences among BZs are of little clinical significance in the appearance of withdrawal symptoms and the achievement of complete discontinuation of BZs. They also imply that adverse events associated with discontinuation are manageable and do not constitute consequential barriers to the use of BZs in patients with serious anxiety disorders requiring vigorous, prolonged treatment. 